\title{
ARE NATURAL KINDS REDUCIBLE?
}

\author{
ALEXANDER BIRD
}

University of Bristol

We talk as if there are natural kinds and in particular we quantify over them. We can count the number of elements discovered by Sir Humphrey Davy, or the number of kinds of particle in the standard model. Consequently, it looks at first sight at least, that natural kinds are entities of a sort. In the light of this we may ask certain questions: is the apparent existence of natural kinds real or an illusion? And if real, what sort of entity are natural kinds? Are they sui generis? Or can they be identified with or reduced to some other kind of entity? In this essay I shall look at possible reasons for asserting that either kinds are no sort of entity, or, if they are entities, their existence is equivalent to some fact not involving kinds.

Richard Boyd seems to take the view that the apparent existence of natural kinds is an illusion.

[W] hat is misleading about formulations in terms of the "reality" or "unreality" of kinds, or of the "realism" or "antirealism" about them, is that they wrongly suggest that the issue is one regarding the metaphysical status of the families consisting of the members of the kinds in question - considered by themselves - rather than one regarding the contributions that reference to them may make to accommodation. Issues about "reality" or "realism about" are always issues about accommodation. (Boyd 1999: p.159)

What Boyd means by 'accommodation' is the ability of our theories to support effective inductive inferences. So when we say that a certain natural kind is real, we are not engaging in ontology, discussing the existence of some entity. Instead we are declaring that the use of the natural kind term in our theories is conducive to the inductive power of those theories.

Boyd's statement is perhaps ambiguous on this point, since he talks of 'the contributions that reference to them may make'. If natural kinds can be referred to, then they exist. That suggests a second interpretation of Boyd's comment: that while natural kinds exist, their existence amounts to precisely the fact that the use of natural kinds terms in our theories is inductively fruitful. 
The first interpretation itself may subdivide into two views. The first says that the apparent talk of natural kinds is syntactically misleading. The true logical form of statements containing natural kind terms is not one in which those terms appear as singular terms. The second view concedes that the apparent syntactic form is the true syntactic form, but maintains that this is misleading in so far as the propositions thereby asserted are nonetheless false. That is, we do indeed use singular terms that would refer to entities that are natural kinds, but these terms do not in fact refer (they are empty singular terms), because the entities in question do not exist. Thus we may distinguish three views:

(I) In so far as statements appear to employ natural kind terms as singular terms, those statements are syntactically misleading. The true logical form of those statements is such that they contain no purported referring kind terms.

(II) Statements employing apparently referring natural kind terms are not syntactically misleading, since that is indeed the syntactic function of those terms. But the statements are semantically misleading in the sense that we use them as if they were true, whereas they are in fact false. That is because such terms are empty - there are no entities that are natural kinds to which they can refer.

(III) Natural kind terms are (sometimes) successfully referring terms. So natural kinds do genuinely exist. But the existence of the kinds is in some sense nothing more than the fact that employing the terms in our theories is conductive to inductive success.

While I suspect that Boyd intends (I) with his assertion, there remains a question as to which of these views, if any, is correct.

If we adopt view (I), then we are under some obligation to suggest what the true logical form of a statement containing a natural kind term is. Consider a standard kind of statement about a chemical element, such as:

(S) Mercury is a chemical element, with atomic number 80 , and is among the transition elements; it has a melting point of $234 \mathrm{~K}$, a boiling point of $623 \mathrm{~K}$, and a density of $13.5 \mathrm{~g} . \mathrm{cm}^{-3}$. 
How should we eliminate the use of the apparently singular, referring term, 'mercury'? A natural alternative to suggest is that statements such as (S) are disguised universal quantifications. Instead of a natural kind referring expression we have a natural kind predicate, so that in general, statements of the (apparent) form:

$$
\text { (S0) } \Phi(K)
$$

should be given reducing equivalents of the form:

$$
\text { (R0) } \forall x(K x \rightarrow \Phi x) \text {. }
$$

Let us call the claim under consideration, that every statement of the form (S0) has an equivalent of the form (R0), the 'reducibility claim'. Thus, according to the reducibility claim, the statement:

(S1) Mercury boils at 623K,

which is a component of $(\mathrm{S})$, receives the reduction:

(R1) $\forall x(x$ is a sample of mercury $\rightarrow x$ boils at $623 \mathrm{~K}){ }^{1}$

It is worth mentioning, however, that there are technical reasons why (RI) cannot be equivalent to (S1). One is the phenomenon of superheating. Superheating, which is not uncommon with water heated in a microwave, occurs when a sample of a liquid is heated above the boiling point for that substance. Now this might be thought to be merely a technical quibble. Perhaps the 'correct' replacement for (R1) is something like:

(R1') $\forall x(x$ is a sample of mercury $\rightarrow$ ( $x$ is in a container with sufficient nucleation sites and at a pressure of 1 bar $\rightarrow x$ boils at $630 \mathrm{~K})$ ).

The additional detail that (R1') contains, consists of additional facts that can be known only from a knowledge of the physics of boiling. Consequently the transition from (S1) to (R1') cannot be a purely syntactic trans-

\footnotetext{
${ }^{1}$ One might wonder whether it is strictly true that 'mercury has a boiling of point of $623 \mathrm{~K}$ ' and 'mercury boils at $623 \mathrm{~K}$ ' mean exactly the same. If not, the reductive account here under consideration is in even more difficulty.
} 
formation. Thus, not only is the reducibility claim strictly false, it does not appear as if any other general description of an appropriate syntactic reformulation is viable.

Even if we can give (S1) a fairly obvious reductive equivalent, it is less clear that the same is true for:

(S2) Mercury is a transition element.

This statement refers to the position of mercury within the periodic table of elements. One plausible way of understanding (S2) is as asserting of one kind, mercury, that it belongs to a higher-order kind, the transition elements. The higher-order kind is a kind whose instances are themselves kinds. A higher-order kind is thus a different sort of thing from just a more inclusive kind. ${ }^{2}$ According to the reducibility claim, the reducing equivalent to (S2) is:

(R2) $\forall x(x$ is a sample of mercury $\rightarrow x$ is a transition element).

But that is clearly false, since samples of stuff do not have positions in the periodic table. The transition elements are also known as the 'd-block elements', because they are those elements whose atoms have an incomplete $d$ sub-shell. So instead of (R2) one might try:

(R2') $\forall x(x$ is an atom of mercury $\rightarrow x$ has an incomplete $\mathrm{d}$ subshell).

But the proposal that (R2') is the correct reduction for (S2) is open to two objections. First, the content of (R2') cannot be analytically equivalent to (S2) since it is an a posteriori discovery that the transition elements are those whose atoms have an in- complete $d$ sub-shell. Secondly, the form of (R2') shows a significant deviation from (R0), because the quantification in (R0) is over samples of the kind, where as the quantification in (R2') is over atoms of the kind. Thus the reducibility claim fails if it is considered as a general recipe for reduction. This objection and that of the preceding

\footnotetext{
${ }^{2}$ A set theoretic analogy is the distinction between set $A$ having set $B$ as a member, $B \in A$, and set $A$ being a superset of $B, B \subset A$. However, a more appropriate analogy might be with the theory of types, in which case the claim that (S0), when $\Phi$ is a higher-order kind predicate, always has an equivalent (R0), finds its analogue in the much-reviled axiom of reducibility.
} 
paragraph suggest that we are unable to find reductions that are systematic, and that one reason for this is the fact that the best reductions require the auxiliary use of a posteriori knowledge specific to the nature of the predicate ' $\Phi$ '.

Even if we accept that the reductions provided by (R1') and (R2') are individually unproblematic, a further worry arises from the fact we need a form of reduction that will work simultaneously for supposedly different uses of the kind term. We have seen that the reductions of (S1) and (S2) have different forms, one involving quantification over samples, and the other over atoms. Now let us return to (S). After the semi-colon we have assertions about mercury whose reductions must be in terms of samples of mercury, whereas before the semi-colon we have assertions which can only be reduced to assertions about atoms of mercury. The semi-colon itself must be understood as a conjunction, as is shown by the anaphoric 'it', immediately following the semi-colon. 'Mercury is a transition element with a boiling point of $623 \mathrm{~K}$ ' is perfectly well-formed. ${ }^{3}$ But there is no way of providing a reduction for this sentence, since there is no domain of quantification that it suitable for both predicates simultaneously.

It must be concluded, therefore, that (I) in Section 1 is not a viable option. Which is to say that we must understand natural kind terms as syntactically genuine referring terms. That conclusion is consistent with their in fact failing to refer. And so the next option to consider is that natural kind terms are empty singular terms.

There are natural kind terms that are, we can all agree, empty: 'phlogiston', 'caloric', 'celestial sphere', 'quintessence', 'N-ray'. Option (II) proposes that all natural kind terms are like these. However, the reason why we are convinced that the terms mentioned fail to refer is that the scientific theories hypothesizing the existence of the kinds have been shown to be false. That clearly does not apply across the board. The philosophy of mathematics provides an analogy: we have specific reasons for thinking that certain mathematical singular terms fail to refer, e.g. 'the largest prime number'.

\footnotetext{
${ }^{3}$ If in doubt consider the following statement: 'With a boiling point of $623 \mathrm{~K}$ and low vapour pressure, mercury is a vapour at a lower temperature than any other d-block element, and as such is a suitable substance for the principal component of flourescent lamps.'
} 
Usually, as in this case, the reason is a proof that the corresponding entity cannot exist. But we may also have general reasons for thinking that none of our mathematical terms refer: e.g. metaphysical arguments that mathematical entities could not exist, or that if they do exist, we cannot succeed in referring to them (for example, because we cannot interact with them causally).

Are there any arguments parallel to the mathematical ones, for thinking that singular natural kind terms must fail to refer? Here the burden is on those who wish to make such an argument. The obvious analogues to the mathematical parallel do not furnish such reasons. For example, while it may be true that mathematical entities, if they exist, are causally inert, the same, it would appear, is not true for natural kinds. And in many cases at least, ostensive definition of a kind term is paradigmatic of reference fixing through causal interaction.

However, matters may not be so clear. What are natural kinds? Are they particulars, or universals, sets, sui generis entities? Until we can answer questions of this sort, we are not in a position to be confident either way as regards our ability to interact with kinds causally or to refer to them. If we hold, as Quine (1969) did, that kinds are the sets of their members, then they are abstract entities of a sort similar to mathematical ones. They may then face the same disabilities as such entities when it comes to causal interaction and reference (though we may also note Penelope Maddy's (1990) argument that impure sets can be causal in virtue of the causal efficacy of their members). If kinds are universals, then we are in the arena of the debate between realists and nominalists, given that one argument of the nominalists is that universals are beyond cause and reference. It would be unproductive to pursue this issue further in the absence of further research on the nature of natural kinds. While there has been much consideration of whether there are natural classifications of the world, little has been written on the topic of what, if anything, natural kinds are. (The exception to this being the debate over whether species are individuals or kinds.)

Thus the position for kinds is different from that for mathematical entities. In the case of the latter, we can be reasonably confident that if they exist at all, they are abstract objects. The issues concerning the (im)possibility of reference to such objects arises immediately. In the case of kinds, matters are different; we do not know what such entities would be, and so we do not know whether parallel concerns arise or not. However, when we consider various proposals for the nature of natural kinds, we will 
be able to see whether such a proposal does raise such worries (as does the proposal that kinds are sets). Consequently one of the considerations in play when assessing any such proposal will be whether or not it leads to problems of this sort. That it does so would count against the proposal. It would be premature, therefore, to predict that our best theory of natural kinds must imply that kind terms all fail to refer. On the contrary, there is a good chance that our best theory will not have that implication, simply because not having that implication will be one criterion of being a good theory. As a result, I think we are under no current obligation to regard attempts to refer to natural kinds as failing systematically.

4

The final option we must consider, (III), is that while natural kind terms do refer, their success in so doing is in some sense a reflection of facts in which natural kinds take no part. Again we may turn to a mathematical analogue. According to the neo-Fregean (neo-logicist) position promoted by Wright (1983) and Hale (1987), statements about the identity of numbers are equivalent to statements about the equinumerosity of concepts, encapsulated in Hume's principle:

(N) the number of $F \mathrm{~s}=$ the number of $G \mathrm{~s}$ iff the $F \mathrm{~s}$ and the $G$ s may be put into 1-1 correlation.

Hume's principle does not provide a means of eliminating use of numerical referring expressions, although it does allow us to prove Peano's postulates, with the consequence that the theorems of Peano arithmetic are deducible from second order logic plus $(\mathrm{N})$. Since the numerical expressions cannot be eliminated, $(\mathrm{N})$ does not strictly provide for any kind of reduction - it is more a matter of deflating the commitment to kinds. It would thus be wrong to think of $(\mathrm{N})$ as implying that 'the number of $F \mathrm{~s}=$ the number of $G \mathrm{~s}$ ' is a notational variant on 'the $F \mathrm{~s}$ and the $G$ s may be put into 1-1 correlation'. As a result we must regard 'the number of $F \mathrm{~s}$ ' as a genuine referring expression, and 'the number of $F \mathrm{~s}=$ the number of $G \mathrm{~s}$ ' as a genuine identity claim. The truth of $(\mathrm{N})$ guarantees that some such identity claims are true and so that some numerical referring expressions succeed in referring. 
Could we employ a similar approach for natural kinds? Just as facts about the identity of numbers reflect or are shadows of (but are not identical to) facts about equinumerosity, facts about natural kinds would reflect facts concerning some equivalence relation on instances of a kind. For example, some kind of similarity relation might ground identity of natural kinds. Putnam's (1973: pp.702-703) discussion of water suggests something along those lines:

(K) The kind to which a belongs = the kind to which $\mathbf{b}$ belongs iff $\mathbf{a}$ and $\mathbf{b}$ are related by a same-kind relation. ${ }^{4}$

The 'same-kind' relation must be one that is not equivalent to 'belongs to the same kind as', for then $(\mathrm{K})$ would be uninformatively tautologous. The problem is to find something that will fill this role. It is a problem for two reasons. First, it is unclear that any relation will do for all natural kinds. For chemical kinds one might think that sameness of micro-constitution will do. But this will not work for biological kinds. Secondly, if kinds are defined by this same-kind relation, that would imply that there is at most only one kind to which any individual can belong, whereas there can be nested kinds, and even cross-cutting ones.

One response to such problems is to regard $(\mathrm{K})$ as providing a schema for more specific abstraction principles. So:

(C) The chemical compound of which $\mathbf{a}$ is an instance = the chemical compound of which $\mathbf{b}$ is an instance iff $\mathbf{a}$ and $\mathbf{b}$ share the same molecular structure,

and:

(B) The species to which a belongs = the species to which $\mathbf{b}$ belongs iff $\mathbf{a}$ and $\mathbf{b}$ are both members of the same breeding population,

might be first passes at abstraction principles in chemistry and biology. Furthermore, different equivalence relations from the same science may define different kinds, allowing for one item to be a member of several different kinds.

\footnotetext{
${ }^{4}$ Putnam says 'same liquid' but that clearly will not do, since a phase difference does not imply a kind difference.
} 
The last suggestion does raise a possible problem in the opposite direction, that we might have too many kinds. Does any equivalence relation on natural objects define a natural kind? Presumably not; this is the analogue to Alan Weir's (2003) 'embarrassment of riches' problem for neoFregeanism. To avoid this problem scheme (K) must be supplemented by a principle which states which equivalence relations may legitimately be substituted for the 'same-kind relation' of $(\mathrm{K})$. As the quotation from Boyd suggests, we want the use of our kind terms to reflect and underwrite our inductive and explanatory practices. So the appropriate principle is one that restricts the substitutable relations to those that will give inductive and explanatory power to kinds. (C) is legitimate, for example, because molecular structure is inductively and explanatorily powerful in chemistry. There will be natural similarities between objects, such as colour, which will support some inductions, but not enough to base a natural kind. Thus the concept of natural kind captured by $(\mathrm{K})$ will be vague.

We have considered three ways in which natural kinds might, in some sense, be reduced. The first suggested that statements containing singular natural kind terms are syntactically misleading and that such statements are equivalent to statements not containing such terms. Such a suggestion fails, since their is no systematic way of eliminating the relevant terms for all statements, including complex ones in which several different predications are made with the same natural kind term in subject position. The second possibility was an elimination of natural kinds, considering all natural kind terms to be semantically empty. Such a view can be neither substantiated nor refuted without further investigation of what sort of entities natural kinds would be if they do exist. But as it stands, natural kinds do seem to be capable of being referred to and any account of their existence that denied this would be prima facie implausible. Finally we considered a neoFregean approach. This seems the most promising avenue. But it is also the least reductive. On this view, natural kinds do exist. It is reductive only in that there is a conceptual tie between facts about the identity of kinds and facts about equivalence relations among their instances. Furthermore, such a view is also consistent with a more robust view of what natural kind existence is. It is also consistent with natural kind essentialism. As such the neo-Fregean view offers only the weakest kind of support for a reductive 
view. That is, it is consistent with taking the view that kind identity is analytically equivalent to or in some sense constituted by the equivalence relation among kind-instances; in that sense it deflates the existence of kinds. Can we make sense of this in a way that will satisfy the reductionist impulse behind comments such as Boyd's? In the philosophy of mathematics the status of $(\mathrm{N})$ in this regard is a matter of debate (see e.g. Boolos 1987, 1990; Wright 2001). Given the falsity of option (I) and the prima facie implausibility of (II), we have to regard the left hand sides of instances of (K) as successfully referring to entities that are kinds. But how then can the left hand sides be mere reflections of the contents of the right hand sides, in which no reference to kinds is made? On the other hand if the right hand sides have a hidden commitment to the existence of kinds, then although the left hand sides may be equivalent (making the commitment to kinds explicit), (K) cannot now be used to deflate the existence of kinds to mere reflection of inductive similarity among instances. In the light of this, it is uncertain even that the deflation of kinds via $(\mathrm{K})$ is successful.

\section{REFERENCES}

Boolos, G. 1987. The Consistency of Frege's Foundations of Arithmetic. In J. Thomson (Ed.), On Being and Saying: Essays in Honor of Richard Cartwright, pp.320. Cambridge, MA: MIT Press.

Boolos, G. 1990. The Standard of Equality of Numbers. In G. Boolos (Ed.), Meaning and Method: Essays in Honor of Hilary Putnam, pp.261-277. Cambridge: Cambridge University Press.

Boyd, R. 1999. Homeostasis, Species, and Higher Taxa. In R.A. Wilson (Ed.), Species: New Interdisciplinary Essays, pp.141-185. Cambridge: MIT Press.

Hale, B. 1987. Abstract Objects. Oxford: Blackwell.

Maddy, P. 1990. Realism in Mathematics. Oxford: Oxford University Press.

Putnam, H. 1973. Meaning and Reference. Journal of Philosophy 70: pp.699-711.

Quine, W.V. 1969. Natural Kinds. In Ontological Relativity and Other Essays, pp.114-138. Columbia University Press.

Weir, A. 2003. Neo-Fregeanism: an Embarrassment of Riches. Notre Dame Journal of Formal Logic 44: pp.13-48.

Wright, C. 1983. Frege's Conception of Numbers as Objects. Aberdeen: Aberdeen University Press.

Wright, C. 2001. Is Hume's Principle Analytic? In B. Hale and C. Wright (Eds.), The Reason's Proper Study, pp.307-333. Oxford: Oxford University Press. 